\title{
Supervisory Control of Multiple Social Robots for Navigation
}

\author{
Kuanhao Zheng ${ }^{1,2} \quad$ Dylan F. Glas ${ }^{1,2} \quad$ Takayuki Kanda $^{1}$ \\ 1. Intelligent Robotics and Communication Laboratories \\ ATR \\ Keihanna Science City, Kyoto, 619-0288 Japan \\ Hiroshi Ishiguro $^{1,2} \quad$ Norihiro Hagita $^{1}$ \\ 2. Intelligent Robotics Laboratory \\ Osaka University \\ Toyonaka, Osaka, 560-8531 Japan
}

\{tei, dylan, kanda, ishiguro, hagita\}@atr.jp

\begin{abstract}
This paper presents a human study and system implementation for the supervisory control of multiple social robots for navigational tasks. We studied the acceptable range of speed for robots interacting with people through navigation, and we discovered that entertaining people by speaking during navigation can increase people's tolerance toward robots' slow locomotion speed. Based on these results and using a robot safety model developed to ensure safety of robots during navigation, we implemented an algorithm which can proactively adjust robot behaviors during navigation to improve the performance of a human-robot team consisting of a single operator and multiple mobile social robots. Finally, we implemented a semi-autonomous robot system and conducted experiments in a shopping mall to verify the effectiveness of our proposed methods in a real-world environment.
\end{abstract}

Index Terms-Human-robot interaction, social robots, navigation, supervisory control

\section{I.INTRODUCTION}

Research of human-robot interaction using mobile robots has been conducted in a variety of environments, such as shopping areas [1-3] and museums [4-6], suggesting great potential for mobile social robots as real-world service providers in the near future. By leveraging their navigational ability, social robots can provide useful services such as route guidance [7], shopping support [8], and baggage carrying [9], by moving together with customers in shopping areas.

However, it is still impossible to realize fully autonomous mobile robots due to safety considerations and immaturity of current technology. Supervisory control [10] is an effective way of ensuring robot safety and compensating for technology limitations, wherein a human operator controls the robots when there are potential dangers or failures of autonomy during navigation. Because large proportion of tasks can be conducted autonomously, it is possible to enable a single operator to supervise multiple robots by only controlling the robots when necessary, which can extend human capabilities and improve system efficiency [11].

A major challenge in realizing supervisory control of multiple semi-autonomous robots is to keep the performance of the robots above some acceptable level, given that the operator has limited time to pay attention to each robot. For example, if the operator's attention is focused too much on a single robot, then the other robots may be overlooked and operating in degraded states for a long time, which may eventually lead robots to dangerous states or decrease the total performance of the robot team. For robots conducting social interactions with people, degraded responses by the robots can cause frustration and a negative impression toward the robots [12].

In this study, we have developed models for the performance of robots in navigational tasks from the perspective of service recipients. We have also developed a technique to anticipate the performance of the overall robot team and proactively control the navigational behaviors of robots in order to maximize total effectiveness in satisfying the customers. These models also enable us to simulate the performance of human-robot teams under different sets of conditions, providing a way to predict how many robots can be supervised by one operator. To demonstrate these techniques, we implemented a system consisting of one operator and four semi-autonomous mobile social robots to provide services by navigating in a shopping mall with customers (Fig. 1).

In the following sections, we will first compare this study with related work. Then, we introduce our interaction models for customer and robot, which we used to develop a scheduling algorithm for operation. Finally, we present a field experiment conducted as a proof-of-concept of the model and system developed in this study.

\section{RELATED WORK}

An important concept related to human-multi-robot system is "fan-out" $[13,14]$, wherein the number of robots that one operator can control can be predicted by measuring neglect tolerance and interface efficiency of the interaction schemes employed by the human-robot team. However, those studies did not explore the idea of neglect tolerance in the context of

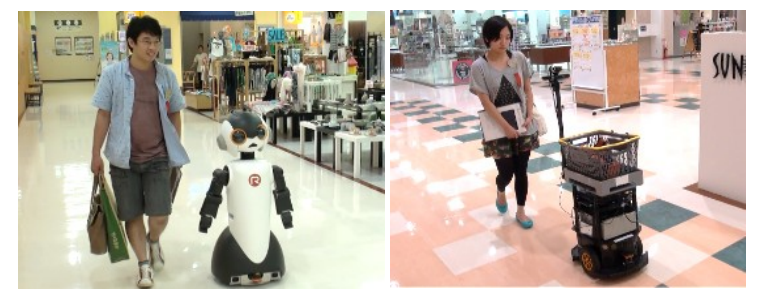

Fig. 1. A humanoid robot and a cart-type robot walking with customers in a shopping mall 


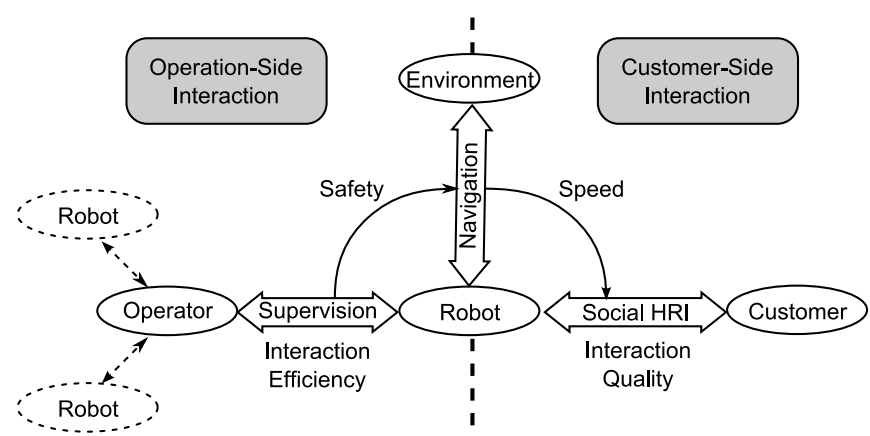

Fig. 2. Interaction model overview

social robots that conduct interactions with people.

A study by Kaber et al. [15] suggests that user perceptions of social etiquette and degree of anthropomorphism are critical in social robot design. In previous studies [16-18], we observed neglect tolerance when the robots conducted conversational interactions with people. We found it useful to use "customer satisfaction," that is, subjective satisfaction with the robot's service as reported by service recipients, as a metric for robot performance. This study extends that previous work into the realm of navigational interactions. We will model neglect tolerance by considering the speed of the robot and whether the robot is speaking while in motion, which are revealed to have important effects on the quality of navigational interactions evaluated from the customer's perspective.

Other studies $[11,19]$ show that the efficiency with which the operator's attention is allocated among the robots is critical to the team's success. Those studies model interactions as stochastic processes, and compute an optimal operator attention allocation scheme assuming that changes in the operator allocation scheme would not change the stochastic structure of other processes. This study adopts a similar idea, but differs from the previous algorithm by enabling the robots to adjust behaviors based on anticipation of future operation, which results in dynamically adjustable neglect time. This study shows that our algorithm can improve the team performance compared to algorithms without such dynamic features.

\section{MODEL OVERVIEW}

An overview of the interaction model is shown in Fig. 2, which can be divided into two parts: customer-side interactions and operation-side interactions:

1) Social human-robot interaction through navigation

The focus of this study is on social robots which can interact with people and provide services by navigating in a given environment, such as providing route guidance in a shopping area. We define "customers" in this study to refer to the people who interact with robots as service receivers. This role differs from the role of "operator", who assists the services provided to the customers by supervising the robots.

The performance in navigation directly affects the quality of interaction between robots and customers. Navigational performance can be affected by a number of factors, such as accuracy of localization and path planning, and we focus on the way the robot's speed during navigation affects the perceptions of customers. We find that customers have an acceptable range of speed for robot navigation, and the acceptable range is affected by social interaction with speech. From such findings, we propose an effective method called "proactive speed control" to manage interaction quality during navigation.

2) Supervisory control of multiple robots by the operator

The operator's task in this study is human control of the navigation of multiple robots using a teleoperation interface. Specifically, we use the operator to ensure safety by localizing the robot when potential risk exists due to uncertainties in navigation. We will discuss how localization failures can create safety-critical situations, and we develop an algorithm to estimate this risk during navigation. Based on this, we develop a scheduling algorithm to efficiently allocate the operator to the robots based on risk estimation, and also maximize interaction quality with customers by using proactive speed control based on a model of customer satisfaction.

\section{Customer Model}

In this section, we present our model of customer satisfaction based on navigational performance of the robots. We will propose a method to improve customer satisfaction by adjusting robot velocity in navigation to reduce stopping, and we present a data collection to find out the relationship between velocity and customer satisfaction.

\section{A. Problem Description}

The goal of this study is to enable social robots to satisfy the customers through navigational interactions. Customer satisfaction can be affected by various factors, such as the success of navigation, the smoothness of locomotion, and the social appropriateness of interaction. Among the various factors, we are interested in the delay in navigation caused by failure of automation. We assume the use of semi-autonomous robots which can conduct successful navigation given correct localization and path planning, but which sometimes may stop due to failures in autonomy.

The stopping of robots during navigation will cause negative impressions to customers because the service is interrupted, and the customers have to wait until the service is restarted. In previous studies $[17,18]$ we found that stopping during conversational interactions will cause a drop in customer satisfaction with the service the robot is providing. We believe this will also be true for navigational interactions, and our purpose is to improve customer satisfaction by reducing stopping time during navigation.

\section{B. Proactive Speed Control (PSC)}

Previous studies [16-18] proposed a method to proactively

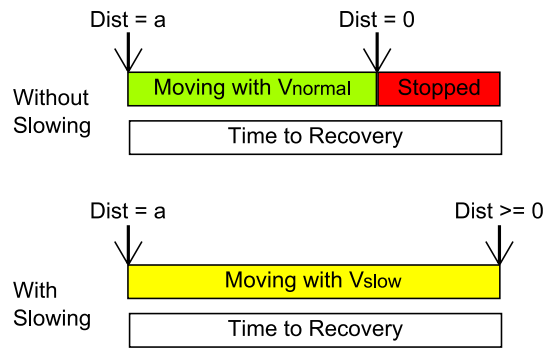

Fig. 3. The strategy of proactive speed control to prevent stopping 
adjust robot behaviors in conversation to improve customer satisfaction by anticipating when the robot will need to interrupt its service to wait for an operator. We borrow the ideas from those studies, and provide a similar strategy called proactive speed control (PSC) to reduce stopping time in navigation by proactively adjusting the robot's speed.

Our strategy to reduce the stopping time in navigation is to use an acceptable slow speed to extend the time to stop when the distance to stop in navigation can be estimated. Fig. 3 illustrates the basic idea of proactive speed control. When the distance to an anticipated failure and the estimated time to recover from that failure are given, stopping is unavoidable when the time to failure based on the current moving speed is shorter than the estimated time to recovery. When using a slower speed, the time to failure will be extended, hence reducing or eliminating stop time. The distance to failure and time to recovery are related to the environment and operation of robots, which will be discussed in the next sections.

Slower speeds are more effective in delaying interruption of service, but speeds that are too slow will also cause customer frustration by delaying the time to reach the goal. Hence, we need to find a proper speed which can be used to reduce stopping while not causing too much frustration. A previous study [20] revealed that individual people have preferable ranges of speed while walking together with a robot. Studies of conversational interactions $[12,17,18]$ have found that the use of speech by robots during degraded states can effectively reduce customer frustration. By combining those ideas, our solution is to choose an acceptable slow speed, and to use speech to increase people's tolerance toward that slow speed.

We conducted a data collection to find out acceptable ranges of speed and whether speech in navigation can effectively increase people's tolerance toward slow speed.

\section{Data Collection}

Twenty people (11 female, 9 male, average age 21.35 , s.d. 2.11) participated as customers in the data collection, most of whom were undergraduate students.

\section{1) Scenario}

To investigate whether different types of services using navigation may affect people's acceptable range of speed, we designed two scenarios, each defining a different navigational task using a different type of robot.

- $\quad$ Route Guidance: In this scenario, we assume the robot provides guidance in a shopping mall by leading customers from a start position to a shop of their choice. We used a humanoid robot as shown in Fig. 1.

- Baggage Carrying: In this scenario, the robot carried the customer's baggage while leading the customer to some goal position. We used a cart type robot which can carry baggage on top of it, as shown in Fig. 1.

Both types of robots have the same range of locomotion speed, and in both scenarios, we let participants follow the robot while walking together.

2) Procedure

We chose a 33-meter straight path in a shopping mall as the navigation path for our data collection. There are two different shops at each end of the path, and each navigational task is started from one end of the path to a shop at the other end. Each participant acted as a customer in both scenarios.

Each scenario contains two sections, one with speech, and the other without speech. In the with-speaking section, the robot spoke predefined phrases about the destination shop or the service it is providing. The speech was one-sided, so that the participant did not need to respond to the speech. In the without-speaking section, the robot moved silently during the whole interaction.

In each section, the robot performed six tasks with the participant using a constant speed during each task among $0.2 \mathrm{~m} / \mathrm{s}, 0.4 \mathrm{~m} / \mathrm{s}, 0.6 \mathrm{~m} / \mathrm{s}, 0.8 \mathrm{~m} / \mathrm{s}, 1.0 \mathrm{~m} / \mathrm{s}$ and $1.2 \mathrm{~m} / \mathrm{s}$ in either increasing or decreasing order. The start and goal positions are switched after each task. After each task, the participants evaluated their satisfaction toward the service with a number ranging from -5 to 5 , where the minimum and maximum values represent lowest and highest satisfaction. The zero value was explained to the participants as their acceptance level, below which it means they would consider the service to be unacceptable. The orders of scenarios, sections, and speeds were counter-balanced among participants.

\section{3) Result}

Fig. 4 shows the data collection result of mean satisfaction evaluated from participants for each scenario. The data collection result shows that there is an acceptable range of speed with positive satisfaction for each scenario and condition. In the route guidance scenario, the acceptable ranges of speed were $0.4 \mathrm{~m} / \mathrm{s} \sim 1.2 \mathrm{~m} / \mathrm{s}$ in the with-speaking condition, and $0.6 \mathrm{~m} / \mathrm{s} \sim 1.0 \mathrm{~m} / \mathrm{s}$ in the without-speaking condition. In the baggage carrying scenario, the acceptable ranges of speed were

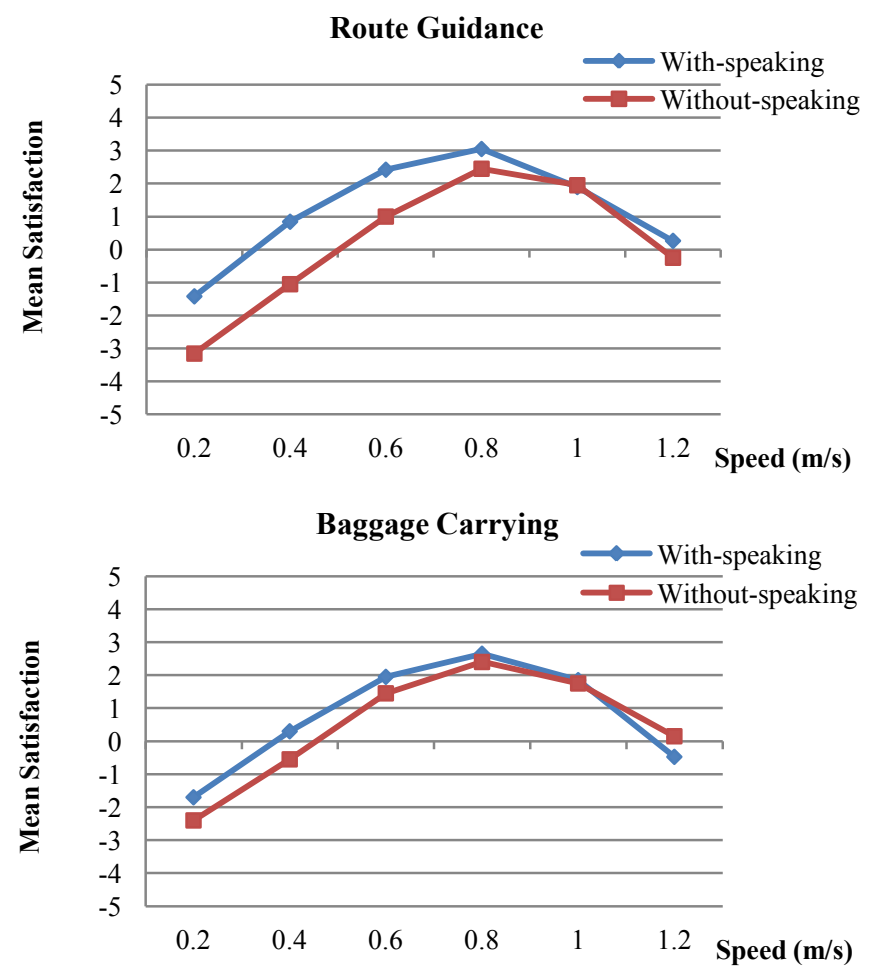

Fig. 4. Data collection result for route guidance and baggage carrying scenarios 
$0.4 \mathrm{~m} / \mathrm{s} \sim 1.0 \mathrm{~m} / \mathrm{s}$ and $0.6 \mathrm{~m} / \mathrm{s} \sim 1.2 \mathrm{~m} / \mathrm{s}$ respectively in withspeaking and without-speaking conditions.

Many people did not like the speed of $1.2 \mathrm{~m} / \mathrm{s}$, although in [21], the walking speed of customers in a shopping mall was measured as $1.12 \mathrm{~m} / \mathrm{s}$ (s.d. 0.16 ), which is closest to the highest speed used in this study. From interviews with some of the participants, we found that typical reasons are as follows:

1. Some participants felt uncomfortable when guided by the robot in such a high speed.

2. One participant said she wanted to glance at some other shops while being guided, but she couldn't do it when walking quickly.

3. Two participants concerned that although they could follow up the robot, maybe some elderly people cannot, so that gave lower score for the high speed.

From the data collection we found the acceptable ranges of speed under different settings, which we use for identifying the minimum acceptable speed for use in our proposed method for reducing stopping time. We also verified that speaking during navigation can extend the lower bound of the acceptable range of speed. These findings will be used in our algorithm for proactively adjusting the robots' speed during navigation, which will be discussed in the next section.

\section{ROBOT SAFETY MODEL}

Safety is a fundamental requirement in robot navigation, particularly for service robots that move among people. In this section, we present a conservative safety mechanism designed to allow safe navigation of unsupervised robots.

\section{A. Forbidden Areas}

The most critical consideration for a robot deployed in a populated social space is safety - safety of people in the environment, safety of the robot, and safety of the environment itself. While techniques for avoiding static and dynamic obstacles have been available for robots for many years, public spaces like shopping malls often include many obstacles that cannot be detected with on-board sensors. Obstacles like glass walls, clothing racks, benches, and "down" escalators can be difficult or impossible to detect using vision or laser scanners.

Aside from physical safety, there are invisible boundaries defined only by business or social convention, such as the boundary of an open shop or a market space. These boundaries must also be respected by robots, as a robot barging into a shop, café, or rest room could be upsetting to people in those spaces.

Together, we define these regions as "forbidden areas," with some examples shown in Fig. 5. These areas cannot be detected by on-board sensors, but entering such areas is unacceptable, as it may break business and social conventions, or cause severe damage to the robot and the surroundings.

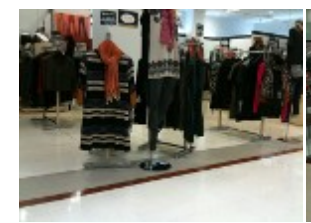

(a)

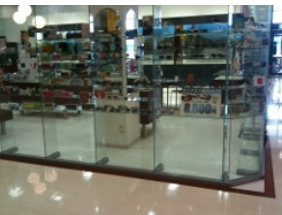

(b)

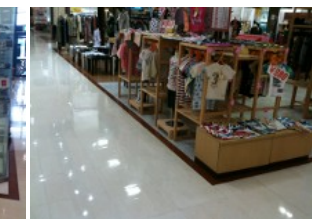

(c)
Fig. 5. Shops with (a) many clothing racks, (b) glass walls, and (c) invisible boundaries
Given a map of the forbidden areas, a robot can avoid these invisible obstacles and social boundaries, but for any localization system, some error is inevitable. If the robot's localization is incorrect, it could result in the robot driving into a glass wall or through a shop display. For this reason, the most critical task of a human supervisor is confirming and correcting the robot's localization.

To address this problem, we first provide the robot with two maps: the first is a map for localization, containing a laser scan map of features in the environment which can be compared with the readings of its on-board laser scanner to localize the robot. The second map defines the boundaries of forbidden areas, including both invisible obstacles and social boundaries.

Regarding robot localization, many common localization techniques exist, but they typically focus on identifying the robot's most likely position, not its position given worst-case localization error. For path planning purposes, the former is useful, but for safety purposes, the latter is necessary.

\section{B. The Safety Polygon}

As there is a wide range of possibilities for the robot's worst-case position, our algorithm estimates the outer boundary of these possibilities, which we will refer to as the "safety polygon," as shown in Fig. 6.

The safety polygon is a powerful tool because it provides a clear criterion for judging whether or not a robot may safely navigate without supervision. As Fig. 6 illustrates, the safety polygon typically grows over time as the robot moves. It is considered safe for the robot to navigate autonomously without supervision until the polygon touches a forbidden area.

At that point, there is a very small, but nonzero, possibility that the robot may actually be at the edge of the forbidden area, and so the robot must be stopped until an operator can confirm its safety.

\section{Risk Estimation}

By stopping the robot whenever the safety polygon touches a forbidden area, safety can be ensured for semi-autonomous robots. However, this comes at a cost. Frequently interrupting a robot service to stop for safety will be frustrating to the users of that service. For the robot to be truly useful, it is necessary to maintain not only safety, but also continuity of service.

To address this issue, our algorithm needs to anticipate when the safety polygon is likely to reach a forbidden area. If the distance to the forbidden area $d_{\text {risk }}$ can be estimated, as shown in Fig. 6, then it is possible to proactively avoid service interruption by prioritizing the allocation of the operator, and by reducing the speed of selected robots to prevent them from stopping, as described in Sec. III-B.
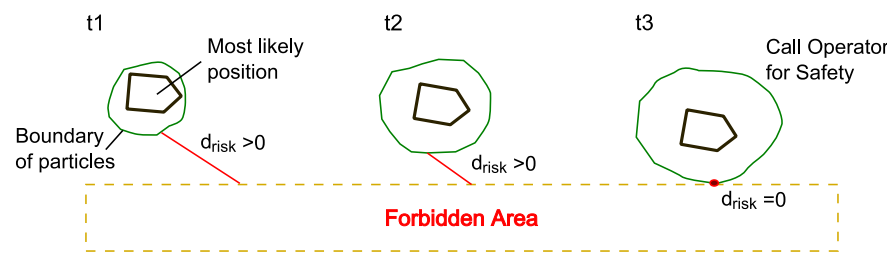

Fig. 6. Basic mechanism of risk estimation algorithm 


\section{Implementation}

A modified particle filter localization algorithm (also known as Monte Carlo Localization [22, 23]) is used as the basic localization technique for our robot. The safety polygon used in our risk estimation is calculated as the outer boundary of particles representing possible position estimates.

The particle filter concurrently performs motion update and measurement update procedures. In motion update, the particles are spread by adding noise representing odometry errors. In the measurement update, the particles are re-sampled based on probabilities calculated by matching laser range finder measurements with a map of features in the environment. Refer to [23] for a detailed implementation of a particle filter.

In typical particle filter localization, the objective is to estimate the robot's highest-likelihood position with high efficiency, which means that even particles with high probability may potentially be removed during re-sampling. To preserve worst-case position estimates, our algorithm is designed differently from a typical particle filter localization algorithm in three respects:

1. In the motion update, we intentionally over-estimate odometry error parameters to spread particles considering large possible odometry errors.

2. In the measurement update, we use a map with only high-confidence features by removing movable features from the map (e.g. benches or clothing racks).

3. Re-sampling is not performed based on relative particle probability. Instead, we only resample particles which absolutely fail to match with the high-confidence map.

The first feature is intended to increase the safety of the worst-case position estimation by spreading the particles in a larger scale, so that a larger odometry error than usual is likely to be captured by a particle. The second feature prevents particles from being erroneously removed due to false mapmatching in changing environments. The third feature is the key to our algorithm, which ensures that all position estimates are maintained, even those with low likelihood - only estimates determined to be actually impossible are removed.

As the result of the algorithm, a set of particles considering the worst-case position estimations is updated during robot navigation, from which we can derive the safety polygon to estimate the risk of robot on entering some forbidden area in worst case. Anticipation of the distance to the forbidden area $d_{\text {risk }}$ is then possible by projecting each particle forward until it intersects a forbidden area.

While no system can truly guarantee $100 \%$ safety, our algorithm allows the designer to directly specify the level of confidence of the safety system, based on how conservatively the odometry parameters are defined.

\section{SCHEDULING ALGORITHM}

Using risk estimation, we can allocate the operator to robots by anticipating each robot's time until stopping, and use the proactive speed control mechanism to adjust a robot's speed when the operator cannot be allocated on time. In this section, we present our scheduling algorithm, and we use simulation to verify its effectiveness under some realistic settings.
SCHEDULING-ALGORITHM $\left(\widetilde{T}_{o p}, v_{\text {normal }}, v_{\text {slow }}\right)$

1: Sort robots by $d_{\text {risk }}$ in increasing order as $R[1 \sim M]$

2: $T_{\text {release }}=$ estimated time to finish current operation

3: for $i: 1 \sim M$

4: $\quad$ if $T_{\text {release }}+\widetilde{T}_{o p} \geq \frac{d_{\text {risk }}[i]}{v_{\text {slow }}}$

5: $\quad v[i]=v_{\text {slow }}$

6: else

7: $\quad v[i]=v_{\text {normal }}$

8: $\quad T_{\text {release }}=T_{\text {release }}+\widetilde{T}_{o p}$

9: if operator is free

10: Assign operator to $R[1]$

\section{A. Algorithm Description}

The algorithm, shown above, takes an estimated operation time $\left(\tilde{T}_{o p}\right)$, a normal moving speed $\left(v_{\text {normal }}\right)$ and a slow speed $\left(v_{\text {slow }}\right)$ used by PSC, and executes the following routine:

First, it creates a queue of robots sorted by the distances to risk in increasing order. $T_{\text {release }}$ is defined as the estimated time before the operator is released to the next robot, which is initialized by the estimated time to finish current operation. In lines 3 to 8 , it iterates through each robot to decide a proper speed based on anticipation of future operation. If the condition in line 4 is satisfied, meaning a robot would be stopped for safety before the operator is available to assist it, the slow speed is assigned in order to delay its time to stop; otherwise the normal speed is assigned. $T_{\text {release }}$ is updated by adding an estimated operation time for one robot after each iteration. Finally, the algorithm assigns the operator to the robot with shortest distance to risk.

The algorithm can be run with high frequency to allocate the operator to the robot with highest risk and assign the proper speed for each robot to avoid service downtime and maximize customer satisfaction by making smooth interactions.

\section{B. Simulation}

In order to test the effectiveness of the scheduling algorithm in managing a human-robot team, we developed a simulation tool which can predict the performance of interactions based on the customer and safety models in this study.

The overall workflow of simulator is illustrated by Fig. 7. It takes the customer model and robot safety model as inputs, and it simulates the timing of interactions by maintaining the states of a simulated operator and a number of simulated robots which are managed by the scheduling algorithm. As the output, the performance of the human-robot team can be calculated based on the timing of simulated interactions.

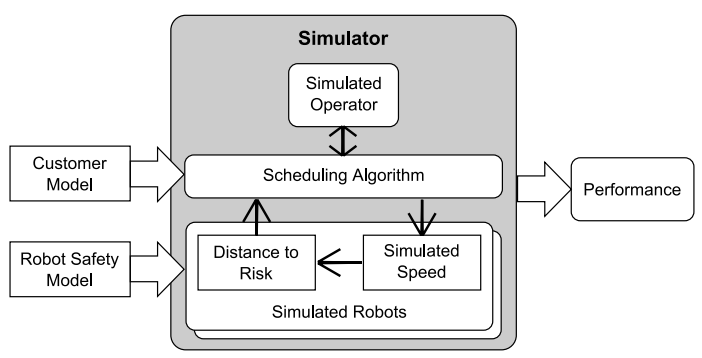

Fig. 7.The overall workflow of the simulation tool 
Using the simulation tool, we analyzed the effect of our scheduling algorithm in improving performance of a humanrobot team consisting of different number of robots. We compared our algorithm with a simple look-ahead algorithm which switches the operator to whichever robot closest to risk without anticipation of operation or proactive speed control.

To get parameters for simulation from the real world, we ran a single robot with risk estimation in some defined paths in a shopping mall, and we measured the distributions of distances the robot travelled while conducting each task and the distribution of estimated distances to risk. An operator who is a researcher in our lab was trained for several hours to use our interface to localize and confirm the robot's position, and we measured operation time during the training. As a result, we measured the distribution of travelling distance for each navigation task as $N\left(19.5,1.0^{2}\right) \mathrm{m}$, the distances to risk as $N\left(16.4,6.2^{2}\right) \mathrm{m}$, and the operation time as $N\left(3.4,2.0^{2}\right) \mathrm{s}$.

Referring to the result of data collection in Section III, we set the normal moving speed as $0.8 \mathrm{~m} / \mathrm{s}$, and slow speed as $0.4 \mathrm{~m} / \mathrm{s}$, because $0.8 \mathrm{~m} / \mathrm{s}$ resulted in highest satisfaction for each type of service, and $0.4 \mathrm{~m} / \mathrm{s}$ is the slowest speed with positive satisfaction when combined with speech.

The performance is defined by Eq. (1), which is the number of acceptable interactions minus the number of unacceptable interactions during the period of all interactions. We defined an interaction as acceptable when the stopping time is less than $10 \%$ of total navigation time; otherwise the interaction is defined as unacceptable.

$$
\text { Performance }=\frac{N_{\text {acceptable }}-N_{\text {unacceptable }}}{\text { Total Time }}
$$

Figure 8 shows the mean performance for different numbers of robots in the two conditions. As the figure shows, the performance when using our scheduling algorithm is superior to simple look-ahead when the number of robots becomes larger than two, and the fan-out, that is, the number of robots providing peak performance, is three.

Figure 9 shows a visualization of simulated timings for three robots under the two conditions, showing how the use of slow speed reduces stopping time. In this simulation, the robots stopped ten times when using the simple look-ahead algorithm, compared with four times when using our algorithm. In this time, 14 services were provided when using the scheduling algorithm, and 13 services were provided using the no lookahead algorithm. Note that the use of PSC does not reduce the number of services, as it only trades slow time for stop time.

The simulation indicated the effectiveness of the scheduling

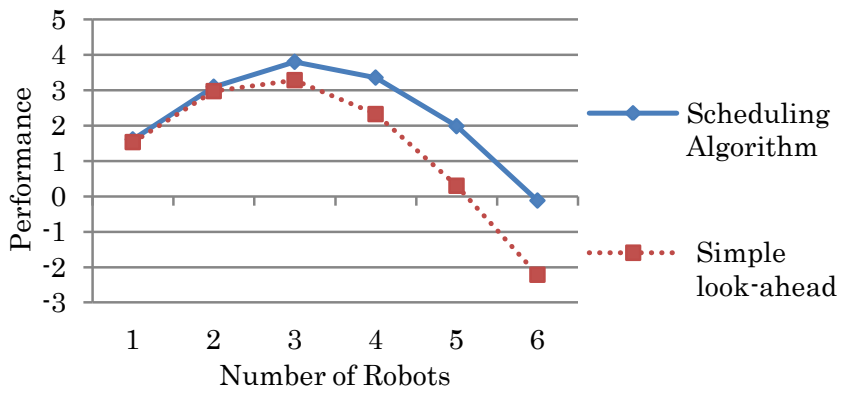

Fig. 8. Mean performance from simulation with different number of robots algorithm, including proactive speed control, using settings measured from the real world.

\section{FIELD EXPERIMENT}

We designed a field experiment to answer two questions:

1. Is the scheduling algorithm effective in reality when used by a human operator as predicted by simulation?

2. Does the scheduling algorithm outperform a human operator who can freely choose which robot to control?

We also wanted to verify whether the risk estimation could keep the robots safe during navigation in a real environment.

\section{A. Settings}

The experiment was conducted in a shopping mall, using two humanoid robots and two cart robots as described in Section III, which conducted either route guidance or baggage carrying services in one of the given paths in Fig. 10. The paths for navigation were surrounded by forbidden areas which the robots are not allowed to enter. As described in Section 4-D, we used both high-confidence and low-confidence features for localization, and only used high-confidence features such as walls and columns for risk estimation.

We decided to use four robots, because based on our previous experiences with conversational robots [16], we expected this would be a feasible target. To produce the condition of continuous interaction with customers for each robot, we recruited eight participants (all male, average age 21.4) to act as customers during the experiment. The operator trained in Sec. VI participated in the experiment.

\section{B. Simulation}

As stated in the settings, our plan for the experiment was to use four robots to simultaneously conduct navigational interactions. But as simulation in Fig. 8 shows, the fan-out under the current settings was only three robots.

From the operator's feedback, we improved the operation interface to make operation more efficient, and as a result, the operation time decreased to $N\left(2.1,1.9^{2}\right) \mathrm{s}$. Using these updated parameters for operation time, we ran the simulation again, which predicted that fan-out would increase to four when using our algorithm. Based on this updated prediction, we carried out our plan of using four robots in the field experiment.

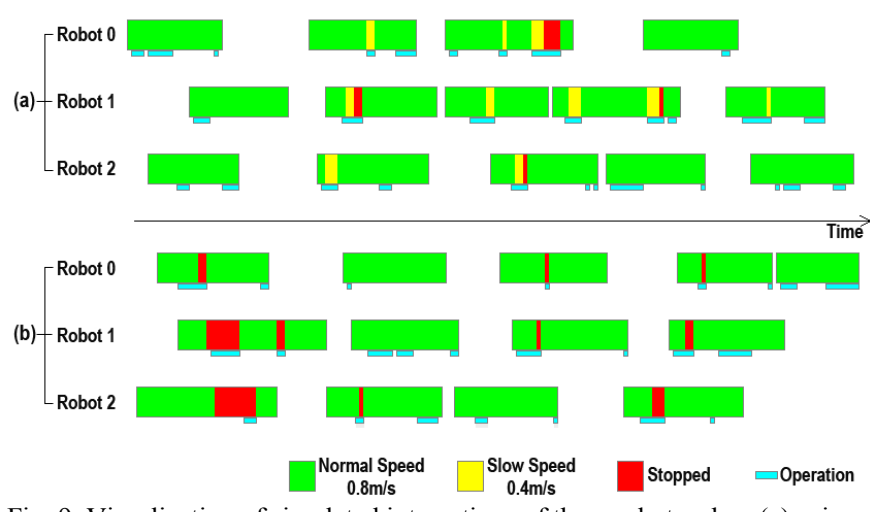

Fig. 9. Visualization of simulated interactions of three robots when (a) using scheduling algorithm and (b) without scheduling algorithm 


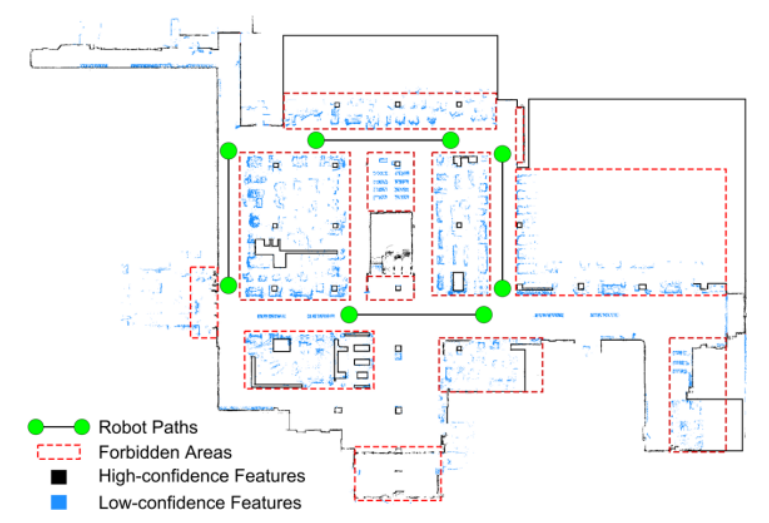

Fig. 10. The environment with robot paths, forbidden areas, high-confidence and low-confidence features

\section{Procedure}

To test the effectiveness of our scheduling algorithm, we defined two conditions: the auto-switching condition, during which we used the scheduling algorithm to automatically allocate the operator and adjust the robots' speed, and the manual-switching condition, wherein we let the operator manually switch among the robots based on observation of each robot's status. Proactive speed control was not used in the manual-switching condition, as the order of operation cannot be anticipated. For each condition, each robot conducted two tasks by going back and forth along a given path with each of the eight participants, carrying baggage or providing guidance. The stopped and slow times of each robot, as well as task duration, were logged during each interaction.

\section{Result}

We conducted the experiment in the shopping mall during a weekday, and Fig. 11 shows the four robots conducting the services simultaneously by walking with participants.

Figure 12 shows the performance of each condition calculated from the logs of robots' speeds, along with the result of simulation conducted prior to the experiment. As the figure shows, performance using the scheduling algorithm was correctly predicted by the simulation. The result also shows that the proposed algorithm performs better than manual switching by the operator.

Table I shows the measured stop, slow times and task duration for each condition. The mean stopping time decreased

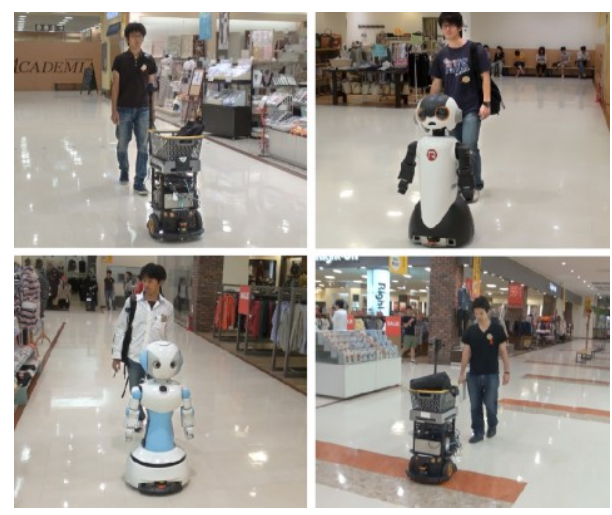

Fig. 11. A scene of four robots moving together with people in the experiment

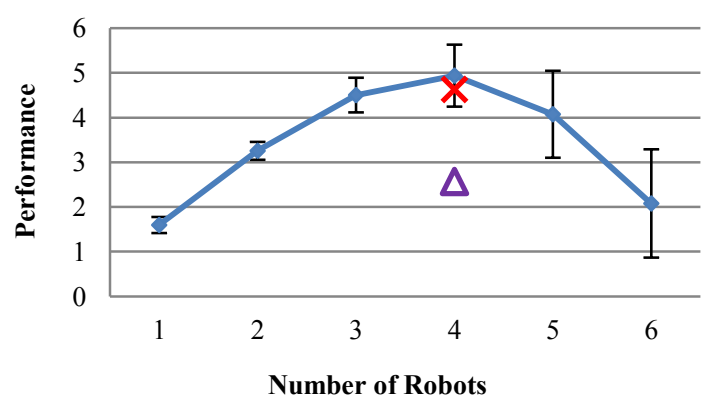

Fig. 12.Predicted performance from simulation $(\diamond)$; actual performance in auto-switching $(\times)$ and manual-switching $(\Delta)$ from the experiment

from 2.23 seconds per interaction in the manual-switching section to 0.52 seconds per interaction in the auto-switching section, and slow speed was used for an average of 0.48 seconds per interaction when using auto-switching. A t-test of stop time between the two conditions showed a significant difference $(\mathrm{t}=2.980, p<.001)$, which indicates that stop time is effectively reduced by auto-switching compared to the manualswitching condition. There was no significant difference between task durations.

After the experiment, we interviewed the participants. Among the eight people, four of them preferred the autoswitching condition. Three of them attributed this to reduced stopping time, and the remaining one said he felt the robot moved more smoothly in that condition. Two participants felt no difference between the conditions, and two participants felt the manual-switching was better because they experienced stopping during the auto-switching condition.

The robots never entered forbidden areas during the total travelled distance of 2438 meters by four robots, which demonstrates the success of the risk estimation algorithm in ensuring safety of mobile robots for navigation.

\section{E. Summary}

Using the interaction models in this study, we successfully predicted the performance of a human-robot team for navigational tasks. The use of PSC improved performance by reducing the stopping time of robots during navigation. We built a working system which enabled single operator to supervise four social robots to perform navigational tasks while ensuring the safety of each robot.

\section{DISCUSSION AND CONCLUSIONS}

\section{A. Discussion}

This study revealed the effectiveness of our predictive model and switching mechanism in managing a team of semiautonomous robots, but several topics remain for future work.

While our performance metric is based on a measurement

TABLE I. TIME MEASUREMENTS FROM THE EXPERIMENT

\begin{tabular}{|l|c|c|c|c|c|c|}
\hline & \multicolumn{2}{|c|}{ Stop Time (s) } & \multicolumn{2}{c|}{ Slow Time (s) } & \multicolumn{2}{c|}{ Task Duration (s) } \\
\cline { 2 - 7 } & Mean & Std. & Mean & Std. & Mean & Std. \\
\hline $\begin{array}{l}\text { Manual- } \\
\text { Switching }\end{array}$ & 2.23 & 3.16 & 0.0 & 0.0 & 38.25 & 3.93 \\
\hline $\begin{array}{l}\text { Auto- } \\
\text { Switching }\end{array}$ & 0.52 & 1.12 & 0.48 & 0.80 & 36.23 & 3.30 \\
\hline
\end{tabular}


of user satisfaction from our data collection, it does not take into account stop time for the robot, frequency of stopping, or the ratio of slow time or stop time to overall service time. Also, to estimate satisfaction in composite tasks, it will be necessary to calibrate the model so satisfaction from navigational and conversational elements of a service can be compared.

One of the contributions of this work is our technique of proactive slow-down to prevent the robot from stopping during a service. However, this technique was seldom used during our field trial because operation time was quite short for our chosen task, while mean time between operations was relatively large. Within these settings, we could achieve a lower rate of stopping by using a more conservative estimate for operation time, which would cause more slow time. In order to properly fine-tune this estimate, a more precise model of people's tolerance toward stopping in navigation is needed.

\section{B. Conclusions}

In this study, we proposed a model for estimating the performance of a team of semi-autonomous service robots supervised by one human operator, conducting human-robot interactions involving locomotion.

To achieve this, we modeled customer satisfaction as a function of the robot's locomotion speed and speaking behavior, and we modeled operator performance based on a navigational risk model for the robot. This modeling made it possible to proactively adjust robot speed to improve overall team performance, by slowing down robots to prevent interruption of service.

We showed the model's effectiveness in predicting team performance in a field trial. The predictive model was also effective as an on-line tool for improving operation efficiency, an approach we hope can be applied to a range of applications for supervisory control of multiple navigational robots.

\section{ACKNOWLEDGMENT}

We would like to thank Satoshi Koizumi for helping to arrange the field experiments. This work was supported by the Ministry of Internal Affairs and Communications of Japan.

\section{REFERENCES}

[1] T. Kanda et al., "An affective guide robot in a shopping mall," HRI2009, pp. 173-180, 2009.

[2] D. F. Glas, T. Kanda, H. Ishiguro and N. Hagita, "Field trial for simultaneous teleoperation of mobile social robots," HRI2009, pp.149-156, 2009.

[3] D. F. Glas, S. Satake, F. Ferreri, T. Kanda and N. Hagita, "The network robot system: enabling social human-robot interaction in public spaces," Journal of Human-Robot Interaction. (to appear)

[4] W. Burgard et al., "The interactive museum tour-guide robot," Proc. National Conf. on Artificial Intelligence, pp. 11-18, 1998.

[5] S. Thrun et al., "Minerva: a second-generation museum tourguide robot," IEEE Int. Conf. on Robotics and Automation, pp. 1999-2005, 1999.

[6] M. Shiomi et al., "Interactive humanoid robots for a science museum,” HRI2006, pp. 305-312, 2006.
[7] Y. Okuno, T. Kanda, M. Imai, H. Ishiguro and N. Hagita, "Providing route directions: design of robot's utterance, gesture, and timing," HRI2009, pp.53-60, 2009.

[8] H.-M. Gross, et al., "Shopbot: progress in developing an interactive mobile shopping assistant for everyday use," IEEE Int. Conf. on Systems, Man, and Cybernetics, pp. 3471-3478, 2008.

[9] Y. Iwamura, M. Shiomi, T. Kanda, H. Ishiguro and N. Hagita, "Do elderly people prefer a conversational humanoid as a shopping assistant partner in supermarkets?" HRI2011, pp. 449456, 2011.

[10] L. Conway, R. A. Volz, and M. W. Walker, "Teleautonomous systems: projecting and coordinating intelligent action at a distance," IEEE Transactions on robotics and automation, vol. 6, No. 2, April 1990.

[11] J. W. Crandall and M. L. Cummings, "Developing performance metrics for supervisory control of multiple robots," HRI2007, pp.33-40, 2007.

[12] T. Shiwa et al., "How quickly should communication robots respond?" HRI2008, pp.153-160, 2008.

[13] D. R. Olsen and M. A. Goodrich, "Metrics for evaluating human-robot interactions", Performance Metrics for Intelligent Systems Workshop, Gaithersburg, Maryland, USA, 2003.

[14] J. W. Crandall, C. W. Nielsen, and M. A. Goodrich, "Towards predicting robot team performance," IEEE Int. Conf. on Systems, Man, and Cybernetics, October 5-8, 2003, pp. 906-911.

[15] T. Zhang, B. Zhu and D. B. Kaber, "Anthropomorphism and social robots," in Human-Computer Etiquette: Cultural Expectations and the Design Implications They Place on Computers and Technology, C. C. Hayes and C. A. Miller, Eds. Auerbach, 2010, pp. 231-259.

[16] D. F.Glas, T. Kanda, H. Ishiguro and N. Hagita, "Simultaneous teleoperation of multiple social robots," HRI2008, pp.311-318, 2008.

[17] K. Zheng, D. F. Glas, T. Kanda, H. Ishiguro and N. Hagita, "How many social robots can one operator control?" HRI2011, pp. 379-386, 2011.

[18] K. Zheng, D. F. Glas, T. Kanda, H. Ishiguro and N. Hagita, "Designing and implementing a human-robot team for social interactions", IEEE Trans. Systems, Man, and Cybernetics Part A. (accepted for publication)

[19] J. W. Crandall, M. L. Cummings, M. Penna and P.M.A. de Jong, "Computing the effects of operator attention allocation in human control of multiple robots," IEEE Transactions on Systems, Man, and Cybernetics - Part A: Systems and Humans, Vol. 41, No. 3, pp. 385-397, May 2011.

[20] E. Sviestins, N. Mitsunaga, T. Kanda, H. Ishiguro and N. Hagita, "Speed adaptation for a robot walking with a human," HRI2007, pp. 349-356, 2007.

[21] H. Kidokoro, T. Kanda, D. Brščić and M. Shiomi, "Will I bother here? - A robot anticipating its influence on pedestrian walking comfort -", HRI2013.

[22] J.-S. Gutmann, W. Burgard, D. Fox and K. Konolige, "An experimental comparison of localization methods", IEEE/RSJ Int. Conf. Intelligent Robots and Systems, pp. 736-743, 1998.

[23] S. Thrun, W. Burgard and D. Fox, Probabilistic Robotics, MIT Press: Cambridge, MA, 2005, pp.250-267. 